\title{
Frontières
}

\section{Le droit de ne pas souffrir}

\section{Simone Plourde}

Volume 17, numéro 2, printemps 2005

Surtout, ne pas souffrir

URI : https://id.erudit.org/iderudit/1073484ar

DOI : https://doi.org/10.7202/1073484ar

Aller au sommaire du numéro

Éditeur(s)

Université du Québec à Montréal

ISSN

1180-3479 (imprimé)

1916-0976 (numérique)

Découvrir la revue

Citer cet article

Plourde, S. (2005). Le droit de ne pas souffrir. Frontières, 17(2), 13-20.

https://doi.org/10.7202/1073484ar

\section{Résumé de l'article}

La solitude de l'être se manifeste tout particulièrement dans le travail, la peine, la souffrance, que le philosophe Emmanuel Levinas rattache à l'engagement de toute personne dans l'existence. Peut-on rêver que la science et la technologie en arrivent à éliminer la souffrance, voire la mort ? Cet article pose la question. Il tente de distinguer souffrance et douleur, développe des considérations sur le rôle et les conséquences de la douleur, sur la revendication de l'individu qui réclame le droit d'être soulagé quand il souffre et sur l'éthique de la compassion.
Ce document est protégé par la loi sur le droit d'auteur. L’utilisation des services d’Érudit (y compris la reproduction) est assujettie à sa politique d'utilisation que vous pouvez consulter en ligne.

https://apropos.erudit.org/fr/usagers/politique-dutilisation/ 
Résumé

La solitude de l'être se manifeste tout particulièrement dans le travail, la peine, la souffrance, que le philosophe Emmanuel Levinas rattache à l'engagement de toute personne dans l'existence. Peut-on rêver que la science et la technologie en arrivent à éliminer la souffrance, voire la mort? Cet article pose la question. II tente de distinguer souffrance et douleur, développe des considérations sur le rôle et les conséquences de la douleur, sur la revendication de l'individu qui réclame le droit d'être soulagé quand il souffre et sur l'éthique de la compassion.

Mots clés: douleur - souffrance alliance thérapeutique - compassion.

\section{Abstract}

Man's solitude is part of his everyday existence. It reveals itself especially in his work, his sadness and in his suffering as pointed out by the philosopher Emmanuel Levinas. Can we hope that one day science and technology will eliminate pain, suffering and even death? This is the question raised by this article. In it, we try to distinguish between pain and suffering; to consider the purpose and the effects of pain; to ask whether or not a person has the right to be treated for pain with everything that is available and to make proposals on the ethics of compassion.

Keywords: pain - suffering therapeutic alliance - compassion.

\section{LE DROIT \\ DE NE PAS SOUFFRIR}

ET C'EST CERTAINEMENT PARCE QUE NOUS SOMMES DES ÊTRES FINIS QUE NOUS SOUFFRONS.

EMMANUEL LEVINAS

\section{Simone Plourde, Ph.D., Groupe de recherche ETHOS, \\ Université du Québec à Rimouski.}

En 1946, le philosophe Emmanuel Levinas fut invité par Jean Wahl, fondateur du Collège Philosophique, à donner à ce collège parisien une série de quatre conférences qui furent publiées, en 1948, sous le titre de Le temps et l'autre. Pour le bénéfice de son auditoire, Levinas explora quelques thèmes axés sur "l'irrémissible poids de l'être». Il démontra que ce poids se fait sentir dans le travail, la peine et la souffrance, phénomènes auxquels se réduit en dernier lieu la solitude de l'individu. Au mot solitude, Levinas conférait un sens précis, ontologique, à savoir: l'intensité du lien de chacun avec soi-même, le définitif de l'identité. Il montrait que l'existant est enchaîné à soi, tant dans l'acte de connaissance que dans l'expérience de la jouissance qui, l'un et l'autre, n'arrivent pas à surmonter la tragédie de la solitude; l'existant est enchaîné à soi dans la douleur du besoin et dans la peine du travail, qui témoignent sans équivoque de l'engagement de l'individu dans l'existence. À tous ses degrés, affirmait Levinas, la souffrance est une exposition directe à l'être, une absence de tout refuge, une impossibilité de se détacher de l'instant de l'existence, de fuir et de reculer (Levinas, 1948, p. 55-56).

Si la douleur et la peine sont à ce point liées à la solitude ontologique, qui peut sans bizarrerie revendiquer le droit de ne pas souffrir? Nos sociétés industrialisées, on le sait, exigent tout de la science. Lucien Sève note fort à propos «qu'au point où en arrive la biomédecine dans ses performances - non seulement curatives mais productives et en quelque sorte démiurgiques, des procréations assistées aux thérapies régénératrices, voire aux futurismes du clonage et de la bionique -, l'opinion tend à être gagnée par une vision entièrement artificialiste de la vie en général, de la vie humaine en particulier où tout en somme se devrait désormais d'être muable, amendable, décidable à gré» (Sève, 2001). La revendication du droit de ne pas souffrir est-elle consécutive à cette vision artificialiste de la vie, appuyée sur une foi illimitée dans la science et les technologies qu'elle suscite? Découle-t-elle d'un optimisme impénitent et de la conviction que, demain, grâce aux recherches et pratiques médicales, la douleur - sinon la mort - sera éliminée?

Il m'a semblé important d'analyser pour lui-même le droit de ne pas souffrir. Cela m'obligera à distinguer douleur et souffrance, avant d'aborder les thèmes connexes du sujet, du rôle et des conséquences de la douleur. Je poursuivrai en examinant deux questions à mon avis inéluctables: la peur de la souffrance qui nous hante et la souffrance d'autrui qui commande l'éthique.

\section{LA DOULEUR ET LA SOUFFRANCE}

Un corps sans douleur est silencieux. Il a l'art de se faire oublier. L'énergie vitale circule en lui sans entrave: il respire, il se meut, il se nourrit, il se repose en temps voulu, il répond bien aux efforts qu'on lui impose. Il est un instrument efficace. Sa mécanique est fiable, elle ne défaille pas. Ses règles propres échappent aux regards. On dit de ce corps qu'il est en santé. "La santé, écrit Hans-Georg Gadamer, est semblable à ce prodige qu'est une harmonie 
forte mais latente. Lorsque nous sommes en bonne santé nous sommes dans un état d'abandon, ouverts à tout ce qui à chaque fois est présent pour nous» (Gadamer, 1998, p. 140). «La santé, affirme Paul Ricoeur, est la modalité propre du vivre bien » (Ricoeur, 1996). Le corps sain se définit par son équilibre et ses pouvoirs.

\section{LA DOULEUR}

En tant que vivant, le corps est un organisme perméable et fragile. Le milieu environnemental l'affecte, des influx nerveux l'assaillent, soit pour l'affaiblir ou le fortifier, le modifiant toujours de quelque façon. Cette vulnérabilité caractérise non seulement le corps, mais marque fortement la dimension spirituelle de l'être humain, faisant de ce dernier un individu, une solitude, un existant unique, complexe, dont l'équilibre et les pouvoirs sont sans cesse menacés. De multiples formes du souffrir assaillent la fragile harmonie, tant du pouvoir-être que du pouvoir-faire. Ce qui fait dire à Paul Ricoeur qu'il y a autant de niveaux de santé qu'il y a de niveaux du souffrir (Ricoeur, 1997, p. 22). Si l'homme sain se définit par son équilibre et ses pouvoirs, l'apparition du souffrir induit une incapacité qui le paralyse dans son agir et dans son être.

La langue française utilise deux mots, douleur et souffrance, pour désigner ce qui fait souffrir. Schématiquement, il semble qu'on réserve plutôt le terme douleur pour signifier ce qui attaque le bien-être du corps ou le physiologique, et qu'on utilise le mot souffrance pour ce qui affecte la partie spirituelle de l'être humain ou le psychologique. Mais la langue quotidienne passe outre à cette distinction: on dira, par exemple, je souffre d'un mal de dent; j'ai la douleur d'avoir perdu un être cher. Le dictionnaire entretient le flou sémantique, en renvoyant l'une à l'autre les deux notions. Le Petit Robert dit de la douleur qu'elle est «une sensation pénible dans un point ou dans une région du corps», mais aussi le «fait de souffrir » à cause d'une affliction, d'un chagrin, d'une déchirure, d'un deuil, d'une peine, notions au moyen desquelles il définit par ailleurs la souffrance elle-même. L'expression «je souffre» n'indique donc nullement la source du mal qui m'affecte. «La souffrance de nos vies, si l'on en croit le psychanalyste Denis Vasse, s'évoque toujours à partir de déchirures ou de dysfonctionnements. Dans le corps ou dans l'esprit. Dans le corps et dans l'esprit» (Vasse, 1983, p. 11). En effet, une individualité sensible est infiniment complexe et mystérieuse; on ne fait guère l'économie du corps dans la souffrance morale, pas plus qu'on ne peut empêcher la douleur physique d'influer sur toutes les dimensions de la souffrance ${ }^{1}$.
L'emploi interchangeable des mots douleur et souffrance témoigne de l'interaction de l'une et l'autre dans l'être humain.

Pour les besoins de ses recherches sur la douleur, l'Association internationale pour l'étude de la douleur a dû définir le terme: «La douleur consiste en une expérience sensorielle et émotionnelle désagréable associée à un dommage tissulaire présent ou potentiel, ou décrite en termes d'un tel dommage $^{2} »$. L'Association rattache d'abord la douleur au dommage qu'elle inflige aux tissus corporels. Mais en insistant sur l'émotion et le désagrément ${ }^{3}$, elle reconnaît que la douleur affecte le patient dans l'ensemble de sa personne. Il serait faux d'affirmer que toute douleur est objective, parce qu'elle est liée à une cause organique. La douleur ne se réduit pas au biologique. Patrick Verspieren met en garde contre une telle réduction qui n'accorde de l'importance « qu'à l'étude des stimuli nociceptifs, des voies de transmission des influx nerveux, des neuro-médiateurs de la douleur..., et à l'emploi des moyens thérapeutiques médicamenteux, chirurgicaux ou électriques agissant à ces différents niveaux» (Verspieren, 1999). Sous l'effet de la douleur, le patient subit contre son gré le refrènement de ses fonctions vitales et, selon la gravité de l'affection, en éprouve tout au moins un désagrément, une frustration. Ce qui fait dire à Peter Kemp que la frustration constitue un degré intermédiaire entre la douleur et la souffrance (Kemp, 1997, p. 161). Parce qu'elle affecte la personnalité du patient, la frustration est en soi une amorce de la souffrance.

\section{LA SOUFFRANCE}

Comme la douleur, la souffrance atteint l'être humain dans son équilibre et ses pouvoirs. Didier Sicard la décrit comme «l'entame angoissante d'un silence organique autrefois rassurant» (Sicard, 1999, p. 161). Maurice Abiven décèle dans la souffrance "l'état douloureux d'une conscience réalisant que ce qu'elle vit, l'état dans lequel elle se trouve provoque limitation de son moi et impuissance» (Abiven, 1994, p. 206). La conscience ajoute une dimension pathétique à la douleur ressentie dans les organes corporels, du fait qu'elle y insère le vecteur temps et anticipe une altération, non seulement des pouvoirs du corps, mais de l'être tout entier. Soudain, la perspective angoissante de la mort hante et menace l'avenir.

Il faut insister sur la structure pluridimensionnelle de la souffrance. La douleur corporelle surgit d'abord comme un avoirmal ponctuel. Quant à la souffrance, elle renvoie à tout ce qui peut faire "souffrir» et tend à s'inscrire dans la durée. Elle évolue en un être-mal ${ }^{4}$ qui affecte le patient dans le plus intime de son humanité, elle l'atteint dans l'ensemble des composantes de sa personne: physiques, psychologiques, affectives, spirituelles, sociales. On appelle couramment souffrance globale la tendance qu'ont ces composantes d'interagir entre elles et de s'intensifier les unes les autres. Les psychanalystes, les psychologues, voire les médecins - ceux qui prennent en compte, non seulement la douleur corporelle, mais aussi cette faille ontologique et sociale creusée par toute souffrance - ne peuvent en nier la globalité. "La souffrance, écrit Denis Vasse, nous situe au carrefour ou à l'entrecroisement de la réalité matérielle, de la réalité psychique et de la réalité du désir. C'est là que, dans le monde symbolique de la parole qui nomme l'homme, se dégage un sujet inscrit au registre d'un réel que toute réalité connote par définition: la réalité matérielle en tant qu'elle l'incarne, la réalité psychique en tant qu'elle le représente et la réalité du désir qui leur échappe» (Vasse, 1983, p. 43-44). En introduisant les notions de désir et de parole, Denis Vasse pénètre dans le registre d'un réel propre à l'être humain, là où s'immisce la pointe acérée de la souffrance. Paul Ricoeur évoque également le monde symbolique de la parole comme lieu de la souffrance, trauma plus vaste que toutes les agressions physiques, lorsqu'il décrit une incapacité d'un type bien particulier, celle qui atteste l'intensité de la souffrance par le mutisme, impuissance qui empêche de se raconter soi-même, «accablé que l'on est par des souvenirs insupportables ou même incompréhensibles ou traumatiques» (Ricoeur, 1997, p. 22).

\section{EXPÉRIENCES HUMAINES}

SANS FRONTIËRE DÉFINIE

Il semble difficile de tracer une frontière invariable entre la douleur et la souffrance ${ }^{5}$. Le philosophe Paul Ricoeur a proposé une distinction qui offre de bons points de repère: "on s'accordera donc pour réserver le terme douleur à des affects ressentis comme localisés dans des organes particuliers du corps ou dans le corps tout entier, et le terme souffrance à des affects ouverts sur la réflexivité, le langage, le rapport à soi, le rapport à autrui, le rapport au sens, au questionnement [...] Mais la douleur pure, purement physique, reste un cas limite, comme l'est peut-être la souffrance supposée purement psychique, laquelle va rarement sans quelque degré de somatisation» (Ricoeur, 1994, p. 59).

Il faut comprendre que douleur et souffrance affectent l'une et l'autre les capacités du patient, tant dans son pouvoir-faire que dans son pouvoir-être. Le sujet qui souffre n'a d'autre choix, pense Ricoeur, «que de persévérer dans le désir d'être et l'effort pour exister en dépit de.... C'est ce "en dépit de..." qui dessine la dernière frontière 


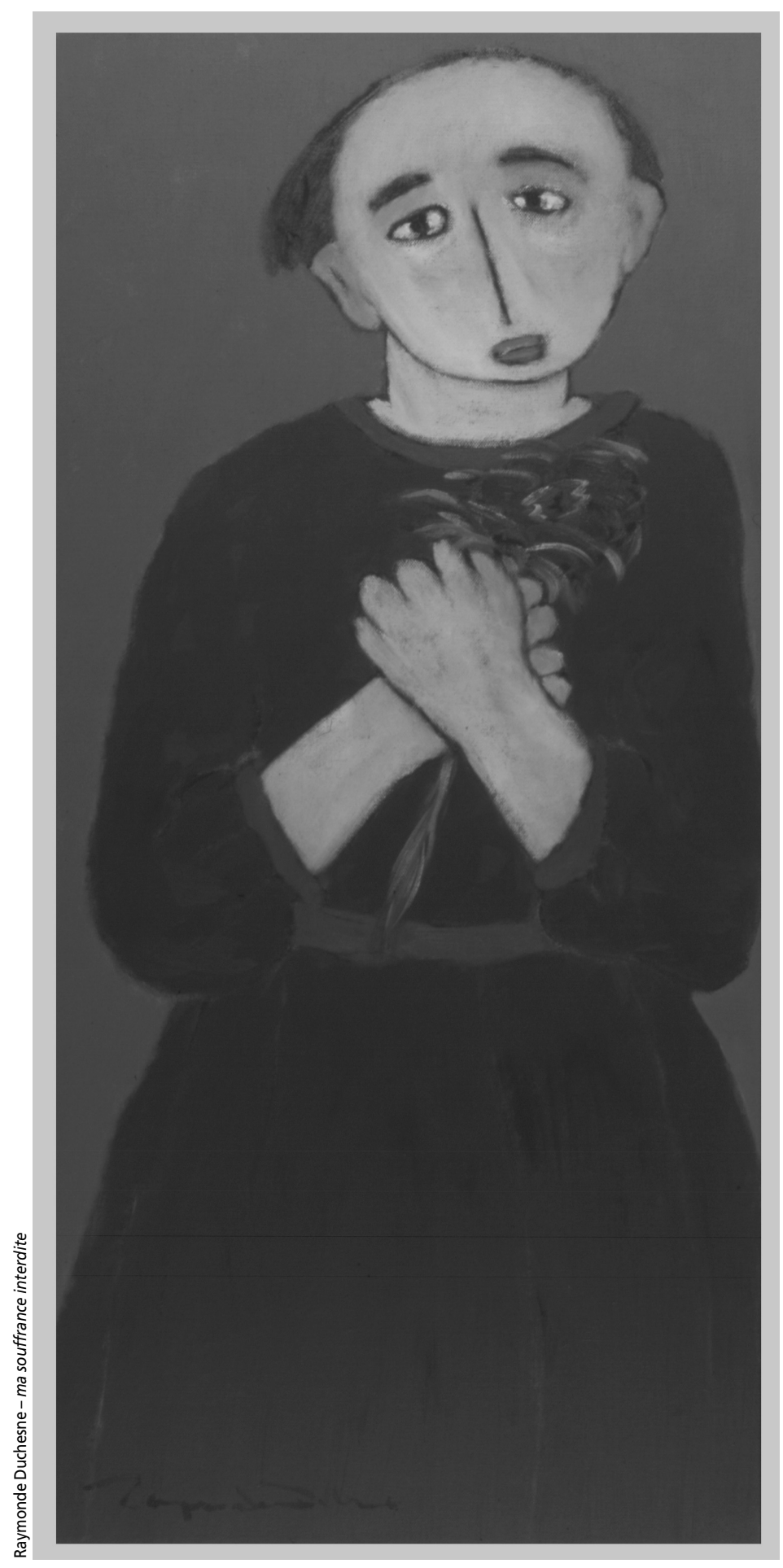

entre la douleur et la souffrance, lors même qu'elles habitent le même corps » (Ibid., p. 69).

Sans frontière définie, les deux réalités affectent différemment le sujet humain aux différents âges de sa vie et selon des modalités qui en modifient la ligne de partage. C'est peut-être l'une des raisons pour lesquelles le philosophe Levinas emploie sans les distinguer les mots douleur et souffrance. Le terme souffrance, plus englobant, semble avoir cependant sa préférence. Toute souffrance, dira-t-il, «est le fait d'être acculé à la vie et à l'être. Dans ce sens, la souffrance est l'impossibilité du néant» (Levinas, 1983, p. 56). Qu'est-ce à dire, sinon que l'existant, dès et tant qu'il est, n'y échappe pas, qu'il est son unicité, sa solitude, qu'il subit la souffrance, cet «événement qu'il n'assume pas, à l'égard duquel

\section{ma souffrance}

\section{interdite}

il est pure passivité, qui est absolument autre, à l'égard duquel il ne peut plus pouvoir» (Ibid., p. 71). Dans une œuvre ultérieure, Emmanuel Levinas précisera que la douleur a partie liée avec la jouissance dont elle est le revers, qu'elle «pointe dans la sensibilité vécue comme bien-être et jouissance ${ }^{6} »$. Les larmes ne trahissentelles pas tant la joie que la douleur?

\section{LE SUJET ET LE RÔLE DE LA DOULEUR}

Je m'attarderai à certains aspects de la douleur physique. Qu'on me permette d'ouvrir une parenthèse et de rappeler que la douleur n'est pas l'apanage de l'être humain. En tant que vivant sensible, l'animal éprouve la douleur. Nos contemporains sont devenus particulièrement préoccupés par la douleur infligée aux animaux, tant par les mauvais traitements qu'on leur fait subir - en particulier ceux exigés par une économie de marché sans conscience ${ }^{7}-$ que par les recherches scientifiques pour lesquelles ils servent de cobayes. On trouve un signe de cette bienveillance inédite à l'égard des animaux dans la Déclaration universelle des droits de l'animal, adoptée par l'Unesco le 15 octobre 1978. Il faut se réjouir de la prise en compte nouvelle d'une réalité qui modifie à l'égard des animaux les comportements humains ${ }^{8}$.

\section{LA PERCEPTION DE LA DOULEUR}

Du côté humain, la douleur apparaît-elle avec la vie? Si l'on ne peut parler de douleur en ce qui concerne le fœtus, celui-ci, comme l'animal, connaîtrait la frustration dès que son système nerveux est suffisamment développé. C'est l'avis de Peter Kemp qui donne en exemple le cas d'un fœtus mal placé dans l'utérus ou celui qui subit les contrecoups du stress maternel (Kemp, 1997, p. 161). Philippe Meyer affirme que la nociception apparaît durant la vie fœtale. Quant au nouveau-né, ajoute-t-il, il peut percevoir la douleur et même avec une grande intensité, dit le Dr Meyer, "par l'immaturité exclusive des messages inhibiteurs de la douleur dans la moelle épinière»(Meyer, 2000, p. 387). Le rapport d'un nourrisson à sa propre douleur est certes très différent de celui des adultes. Il en est de même pour les jeunes enfants. Les jours où ils ne souffrent pas, les enfants hospitalisés mènent une vie bien plus «normale» que les adultes en pareille situation, remarque Jacques Pohier: «il suffit de les voir se promener et jouer dans les couloirs en tirant leur perfusion ou passer un moment à l'école incluse dans le service» (Pohier, 1998, p. 81). Les jeunes enfants n'ont pas la possibilité de prendre du recul par rapport à leur douleur ni celle de la projeter dans l'avenir. Les services pédiatriques connaissent d'ailleurs la difficulté de soulager la douleur ressentie par les enfants malades; leur douleur est souvent sous-estimée parce qu'elle est difficile à repérer et parce que beaucoup d'adultes ont du mal à s'identifier à l'enfant et à faire face à un enfant douloureux.

Le seuil de perception de la douleur varie donc selon les individus et fluctue en fonction de multiples paramètres, entre autres, l'âge, les circonstances, la culture ${ }^{9}$. Ce qui manque à l'enfant malade se trouve chez l'adulte, c'est-à-dire le rapport au temps et l'apprentissage culturel. Les personnes âgées sont généralement affectées sévèrement par la douleur qui affaiblit leur corps et provoque des chutes, des fractures, des troubles du sommeil, des états dépressifs, des pertes d'autonomie, la désorientation, la confusion... Leurs 
douleurs corporelles sont décuplées par la peur, la détresse, la solitude, l'angoisse de la fin de la vie. Nombre de maux somatiques découlent de ces malaises psychologiques (Côté, 2000).

\section{RÔLE ET EFFETS DE LA DOULEUR}

Bien que la neurophysiologie ait accompli des progrès notables depuis les dernières décennies dans la compréhension des mécanismes périphériques et spinaux de la douleur, on ne sait pas très bien expliquer le fait que les récepteurs sensoriels - les nocicepteurs - réagissent aux stimuli potentiellement nuisibles et transmettent au cerveau des informations sensorielles interprétées en termes de douleur. Cette dernière implique certainement une intégration complexe du message sensitif avec d'autres informations émotionnelles et cognitives qui sont activées par l'action du message nociceptif. Une telle constatation a fait dire à Georges Canguilhem, en accord sur ce point avec le célèbre médecin René Leriche: "l'homme fait sa douleur - comme il fait une maladie ou comme il fait son deuil - bien plutôt qu'il ne la reçoit ou ne la subit» (Canguilhem, 1966, p. 56-57). Nous retrouvons dans cette affirmation le croisement déjà signalé de la souffrance avec la douleur.

Afin d'identifier le rôle de la douleur, il faut en distinguer d'abord deux types: la douleur aiguë et la douleur chronique. La douleur aiguë, parce qu'intense et insoutenable, ne dure guère ${ }^{10}$. Elle joue le rôle de système d'alarme: elle est le symptôme d'un dysfonctionnement organique qu'il ne faut pas négliger. L'anthropologue américain Byron Good le dira d'une façon imagée: «La douleur joue un rôle - celui de démon, de monstre tapi au fond du corps, et qui cogne de l'intérieur»(Good, 1998, p. 262). Ce démon est en fait une réaction du système nerveux à une agression interne ou externe du corps. Philippe Meyer décrit scientifiquement la douleur en tant que «l'expression spécifique de l'activation de certaines cellules cérébrales, une manifestation mentale en réponse à un phénomène neuronal». Il ajoute: «On peut envisager que cette motricité réflexe provienne en grande part d'une organisation neuronale spécifique de la moelle épinière. Il s'agit à l'évidence d'un mécanisme mis en place par l'évolution contribuant à la sauvegarde de ceux qui le possèdent» (Meyer, 2000, p. 385). C'est dire que l'absence du mécanisme de la douleur chez une personne est l'équivalent d'un handicap ${ }^{11}$. Georges Canguilhem note que la douleur n'est pas un avertisseur toujours fidèle et toujours infaillible. Cependant, puisqu'il est anormal qu'un être vivant soit indifférent à ses conditions de vie et à la qualité de ses échanges avec le milieu, Canguilhem est

L'IMPOSSIBILITÉ DE FUIR SA DOULEUR ET D'ÉCHAPPER À L'ÉTAU DE SA SOUFFRANCE S'INSCRIT DANS LA PLAINTE, LE CRI, LE SANGLOT, LA BOUCHE BÉE, CES AVEUX D'IMPUISSANCE DEVANT PLUS FORT QUE SOI, LA MORT.

prêt à considérer la douleur comme un sens vital, sans qu'il soit nécessaire d'admettre «qu'elle ait un organe particulier ni qu'elle ait valeur encyclopédique d'information dans l'ordre topographique ou fonctionnel» (Canguilhem, 1966, p. 57).

Il en va un peu autrement de la douleur chronique. Sa chronicité lui retire l'effet protecteur de la douleur-symptôme. Pis encore, son étalement dans la durée rend la douleur destructrice, si elle modifie en profondeur le comportement individuel et social de la personne. La douleur chronique mue souvent en une douleur-maladie, qui évolue pour elle-même au sein du système nerveux. On la dit psychogène ou psychosomatique, si elle devient elle-même une source de pathologie. Lorsqu'elle se présente en fin de vie, elle va souvent en s'amplifiant et mine chez l'être souffrant le désir de vivre; liée à la dégradation du corps qui l'accompagne, elle paraît n'offrir d'issue que dans la mort ${ }^{12}$.

La douleur chronique, terminale ou non, résiste à l'objectivation des tests médicaux standard qui en recherchent la cause pour l'éradiquer par des traitements médicaux. Or, la douleur chronique est rebelle à la localisation; les techniques les plus sophistiquées travaillent en vain à repérer son lieu d'origine, parce qu'elle ne s'inscrit pas nécessairement dans l'un ou l'autre des organes corporels de l'individu, mais se situe à l'interface des champs de sa physiologie, de sa psychologie, et de sa vie en société. Comme le croit Catherine Marquez, «la lésion peut être déjà loin, et la douleur qui l'accompagnait, mémorisée; la limite est parfois ténue entre le souvenir vivant d'une sensation douloureuse et une douleur évoquée par un stimulus actuel» (Marquez, 1994, p. 37).

En conséquence, la charge émotionnelle impliquée dans la douleur chronique en arrive à transformer la conscience et à perturber la vie privée et sociale de l'individu qui se bat contre elle. Toute douleur distrait, fatigue. Quand elle perdure, elle finit par envahir le champ entier de l'esprit. Elle réussit souvent à emmurer intérieurement la personne, à subordonner sa vie aux rythmes de son corps, aux visites chez les médecins et aux séjours en milieu hospitalier. La douleur isole alors le malade chronique, le détourne de ses occupations professionnelles, de ses relations sociales, de ses potentialités d'avenir. Elle démolit son monde.

Le recours aux médecins est une tentative des malades chroniques pour trouver $\mathrm{du}$ soulagement dans la situation traumatisante que certains subissent comme une mise à nu de leur être, blessure intime qu'ils cherchent à masquer ou à nier ${ }^{13}$, en se persuadant que le mal ne se trouve que dans leur corps. Ce serait en effet beaucoup plus simple, s'il en était ainsi. En l'absence de lésion, la douleur chronique n'est pas mesurable et le médecin n'a pas de moyens techniques pour l'évaluer. Les dires des malades en sont la seule preuve et le placent dans une situation inhabituelle d'impuissance. "Comment s'étonner, écrit le Dr Abiven, qu'il [le médecin] ait tendance à minimiser ce symptôme douloureux, voire à le nier? Une prise en charge correcte exige en revanche de lui la reconnaissance de ses limites et la reconnaissance du pouvoir d'appréciation dont dispose son patient»(Abiven, 1994, p. 208). Malheureusement, celui qui est douloureux sans que puisse être repérée, à l'origine de sa douleur, une étiologie organique évidente (maladie, cancer ou traumatisme), fait souvent figure d'imposteur, voire de malade mental. Le personnel soignant ne pense-t-il pas que la médecine ne peut rien pour ces malades dont le mal est jugé imaginaire ${ }^{14}$, sinon de les sécuriser avec «des calmants, ces petits corps pleins de savoir que la société vous donne - sur ordonnance pour avoir la paix ${ }^{15}$ »?

\section{PEUR DE LA SOUFFRANCE}

Considérée du côté du symptôme, la douleur ne renvoie qu'à la maladie dite objective, aux organes corporels, aux diagnostics médicaux et au savoir des médecins. La face subjective de la souffrance s'en trouve occultée. C'est elle maintenant qu'il s'agit de réintroduire.

L'être humain subit le «souffrir» avec une intensité qui lui est propre, parce qu'il possède la conscience la plus fine qui soit parmi les êtres vivants. Grâce à elle, il perçoit non seulement les stimuli adverses qui provoquent la douleur physique, mais il associe la perception douloureuse à un ensemble de représentations mentales complexes, au sein desquelles demeurent vives des émotions, des souvenirs et différentes interprétations personnelles qui sont liées très souvent à la mémoire des douleurs 
passées $^{16}$. Leur présence enfouie dans les replis de la mémoire corporelle et mentale engendre et nourrit la souffrance intime, phénomène qui en laisse plus d'un prisonnier de son passé. Les psychothérapeutes en sont témoins.

Puisqu'elle implique à la fois conscience, mémoire, émotions, la souffrance atteint le psychisme même d'un sujet qui expérimente à la fois l'altération de son corps et de son esprit et la déchirure irréparable dans l'image qu'il s'était faite de soi. Ce sujet se sent mal dans sa peau, s'éprouve contrarié, se voit mené par un chemin qu'il n'a pas voulu, écartelé entre ce qu'il fut, aurait aimé être, et sera désormais. La souffrance initie un nouveau rapport - et combien angoissant - avec le temps ${ }^{17}$, elle "cloue négativement dans le présent ${ }^{18}$ ». En chamboulant l'existence, elle amorce un étrange chapitre de l'histoire personnelle.

En tant que phénomène humain, l'angoisse est un affect, une sorte d'ébranlement interne qui témoigne de la difficulté pour un sujet de s'accorder paisiblement avec soi-même et avec son environnement. "L'angoisse, écrit Philippe Meire, est manifestation de déliaison et de rupture. $\mathrm{Si}$, dans le règne animal, la peur est liée à la menace et au conflit situé à l'extérieur, l'angoisse humaine est liée à la menace et au conflit des représentations que l'homme se crée lui-même. Il craint de ne plus se reconnaître lui-même et de devenir aliéné, c'est-à-dire étranger à lui-même, au point de se sentir mourir » (Meire, 1999).

La souffrance fait peur, d'abord parce qu'elle est un mal-être inconfortable et insupportable $^{19}$ qui peut se prolonger durant des années, mais aussi parce qu'elle se combine avec une situation unique, propre à l'être humain: la conscience de la mort. La souffrance force le patient à devancer l'affrontement avec la fin de l'existence. Elle accentue, pourrait-on dire, la conscience de la vulnérabilité de la vie, dont la multitude des activités quotidiennes réussissent à détourner l'attention. Événement ultime, la mort est ce qui arrive, ce que personne ne peut imaginer, ce qui donne au réel un poids qui pèse lourd sur lui. La souffrance, en tant que mal-être, hérite de ce poids, parce qu'elle conduit au temps où s'affronte la mort. Et la souffrance fait peur autant, sinon plus, que la mort dont chacun cherche à retarder l'imminence par tous les moyens disponibles.

La souffrance est tout entière refus de l'altération, de la déchirure, du mal-être, de la mort. Elle «n'est peut-être même rien d'autre que ce refus de soi: avoir mal, c'est refuser ce qui fait mal, et d'avoir mal ${ }^{20}{ }_{\text {». Elle }}$ est irrationnelle, injustifiable. «Le mal, écrit Xavier Thévenot, est d'abord insensé en tant qu'il est dans sa quiddité non-signification: la souffrance atteint son paroxysme quand il apparaît qu'elle ne sert à rien, qu'elle ne veut rien dire, qu'elle est absurde» (Thévenot, 1997, p. 129). Pourtant, certaines sagesses le stoïcisme, le bouddhisme - ont enseigné à des générations humaines la façon de subir la souffrance, de la transcender et d'y trouver quelque grandeur. Le christianisme l'a considérée comme expiation du mal et moyen de salut.

Le philosophe Gabriel Marcel s'est élevé en son temps contre le dolorisme et contre toute apologie de la souffrance développée par les auteurs de Traités édifiants. À son avis, la souffrance peut toujours être une occasion de raidissement, de contraction, de repliement sur soi, voire de révolte. Elle peut même être entièrement destructrice, maléfique, ce qui s'oppose complètement à la notion édifiante d'après laquelle la souffrance est en elle-même, pour un chrétien, un bien ${ }^{21}$. Mais il arrive que, grâce à quelque sagesse philosophique ou religieuse, quelqu'un puisse trouver un sens à sa souffrance personnelle $^{22}$. La souffrance peut alors devenir un lieu de participation effective à un mystère universel, une participation saisie comme fraternité, comme lien métaphysique $^{23}$. L'ouverture vers le sens, vers la signification, semble dans ce cas, racheter la souffrance sans toutefois la justifier.

\section{PRISE EN CHARGE \\ DE LA SOUFFRANCE D'AUTRUI}

Il existe une autre ouverture vers le sens, c'est celle opérée par l'éthique. Elle s'effectue par la compassion. La vulnérabilité humaine, mise à nu lorsque frappent la douleur et la souffrance, s'extériorise habituellement dans le cri ou la plainte du malade, à moins que la révolte intérieure suscitée par l'intensité du mal-être ne se traduise par le silence, l'enfermement.

Paul Ricoeur décrit ce passage du biologique au symbolique: «Le patient "porte au langage" sa souffrance en la prononçant comme plainte, laquelle comporte une composante descriptive (tel symptôme...) et une composante narrative (un individu enchevêtré dans telles et telles histoires); à son tour la plainte se précise en demande: demande de... (de guérison et, qui sait, de santé, et, pourquoi pas, à l'arrière-plan, d'immortalité) et demande à... adressée comme un appel à tel médecin » (Ricoeur, 1996).

La plainte signifie une impuissance avouée et constitue un appel à l'aide que le psychothérapeute Benjamin Jacobi rattache à l'événement de la naissance, instant où la vulnérabilité est extrême et où la séparation est à la fois reconnue et refusée entre la mère et l'enfant ${ }^{24}$. Toute douleur et toute souffrance raviveraient l'expérience inconsciente de la première rupture. La plainte du patient est celle d'un sujet affronté au désir de vivre et au refus de la mort. «Le cri de la souffrance est la parole d'un sujet qui se cherche dans la déchirure du corps» (Cadoré, 1994, p. 52). Ce cri ouvre un espace où peuvent se rencontrer, dans l'égalité de leur humaine condition, le patient et le médecin. Car, écrit Levinas, la souffrance est un phénomène d'une grande ambiguiité: elle est d'une part «l'inassumable», c'est-àdire passivité extrême, impuissance, abandon, solitude, et d'autre part, «la possibilité d'une entrouverture», un appel originel à l'aide (Levinas, 1984, p. 331). C'est pourquoi «seul un être arrivé à la crispation de sa solitude par la souffrance et à la relation avec la mort se place sur un terrain où la relation avec l'autre devient possible» (Levinas, 1983, p. 64).

La réponse à la plainte est apportée par une prise en charge de la souffrance d'autrui. Si, pour le soulagement de ses douleurs, le patient adulte sollicite en premier lieu une aide médicale, le recours à l'entourage - famille et amis - lui est indispensable pour vivre sa détresse. La responsabilité éthique est dès lors interpellée, car l'éthique ne commence, n'intervient et n'a de sens que dans les rapports entre les personnes et tout ce qui les concerne.

\section{PRISE EN CHARGE MÉDICALE}

Le médecin œuvre au sein d'une institution dont toute douleur, aiguë ou chronique, est la raison d'être, dont l'objectif est de soigner et, si possible, de guérir. Les moyens pour supprimer ou atténuer la douleur s'additionnent au fil de la recherche technoscientifique, qu'ils soient médicamenteux, chirurgicaux, radiothérapiques. Mais derrière le symptôme douloureux se dissimule souvent une souffrance inavouée. Celle-ci demande de l'écoute, la prise en compte de la dimension humaine symbolique au-delà $\mathrm{du}$ somatique. Denis Vasse met en garde contre une manière de soigner «qui évite la parole et qui réduit l'homme à un organisme fonctionnel, sourd et muet» (Vasse, 1983, p. 31). Il avoue s'être souvent demandé si les soins médicaux ne s'employaient pas à faire cesser trop rapidement le cri. «La précipitation de l'urgence, écrit-il, suture souvent la demande. Elle ne laisse pas au cri le temps nécessaire de se convertir en mots " (Vasse, 1983, p. 30). Le psychanalyste rejoint en cela la riche expérience clinique de Didier Sicard: "L'écoute doit être la même pour une rhinopharyngite que pour un diabète grave et c'est parfois derrière cette rhinopharyngite que se cache une autre réalité. Là est réellement le vrai métier du médecin. Ce n'est pas la prescription d'un antibiotique, mais la découverte derrière des symptômes peu graves d'une souffrance qui est parfois la plus importante» (Sicard, 
1999, p. 54). L'écoute active d'un patient confronte chaque médecin à l'essentiel du choix qui est à l'origine de son engagement dans la médecine clinique: venir en aide à l'humanité souffrante par l'écoute et par le soulagement, tant des douleurs que de la souffrance. Ce rôle de bienfaisance ne peut être atteint sans un investissement personnel, souvent difficile, parce qu'il comporte une communion à la souffrance humaine. "Que l'on parle de "sympathie" ou de "compassion", dit un autre médecin, les deux mots disent la même chose: on ne peut aller vers un tel malade sans accepter de s'associer à sa souffrance, sans accepter d'y prendre part, sans souffrir avec lui» (Abiven, 1994, p. 209).

La compassion est l'une des attitudes fondamentales de la responsabilité éthique. Xavier Thévenot la décrit, non comme un état, mais comme «une dialectique d'autre et de même, d'union et de solitude ${ }^{25}$ ». Il définit cette dialectique comme une réaction de souffrance déclenchée dans un être humain par la rencontre avec la souffrance d'un autre. La compassion reconnaît l'intensité du drame d'autrui, épouse sa lutte contre le mal qui l'assaille, lui donne une preuve tangible qu'il n'est pas abandonné, se fait inventive dans le choix des moyens pour soulager sa souffrance. La compassion a ses exigences éthiques: la vérité, la sincérité, l'humilité, qui poussent même le médecin à reconnaître, le cas échéant, son impuissance à guérir ou à soulager, son incapacité à trouver les mots qui amenuisent la détresse exprimée par la plainte.

Le psychothérapeute - et tout particulièrement le psychanalyste - est celui qui, par profession, affronte directement la souffrance psychique d'autrui, que celle-ci s'exprime dans la parole ou par le mutisme: sa compassion s'exerce dans le silence de l'écoute, qui suspend son savoir ou la trop rapide compréhension $\mathrm{du}$ «cas» incarné par son patient. La cure est le fruit d'un travail constant entre deux êtres humains qui met en suspens, dit Denis Vasse, «la prétention hégémonique de notre "moi" » (Vasse, 1983, p. 38). À propos de la compassion, Xavier Thévenot note chez celui qui écoute «un exode souvent douloureux où le désillusionnement s'opère, dévoilant en soi-même, au contact de l'hyper-lucidité de l'autre, des zones bien peu admirables » (Thévenot, 1997, p. 136). En effet, la souffrance unit dans une même aventure le psychothérapeute et son patient, au point que le travail ne peut s'effectuer sans ce que les psychanalystes appellent aujourd'hui une véritable alliance thérapeutique. "Cette alliance, écrit X. Thévenot, est formée entre la partie saine de l'interlocuteur et la partie analysante de celui qui écoute. Elle permet à chacun d'utiliser l'irrationnel de la répéti- tivité transférentielle pour défaire les pièges dans lesquels le désir s'est enfermé provoquant ainsi une souffrance psychique. La compassion peut-elle être le soutien de cette alliance? J'incline à le penser» (Thévenot, 1997, p. 131). La compassion apparaît donc comme une attitude éthique indispensable chez tout thérapeute, pour que l'altération soufferte par le patient trouve le dénouement qui rend la santé.

\section{PRISE EN CHARGE FRATERNELLE}

Les salles d'attente de la consultation sont un lieu où se rencontrent les souffrances humaines les plus diverses. Les patients n'y sont pas toujours seuls: si leur âge ou la gravité de leur état le requièrent, ils sont accompagnés par ceux et celles qui s'inquiètent pour eux, qui peuvent fournir à leur détresse le support de leur amour, de leur amitié ou simplement d'une présence humaine. Leur compassion ne revêt pas la dimension professionnelle de celle des médecins et des thérapeutes. Elle s'inscrit dans le registre de la solidarité humaine. Celle-ci, sensible au cri ou à la plainte de la souffrance, trouve de multiples occasions de se manifester, non seulement envers les membres de la famille immédiate, mais aussi envers des amis, des voisins, des personnes seules, tout être humain, fût-il un inconnu.

Nul mieux qu'Emmanuel Levinas, me semble-t-il, a su rappeler à tous que la souffrance est pour chacun un «malgréla-conscience», un pâtir pur, un non-sens, un excès qui appelle le refus, un fait injustifiable, un cul-de-sac, une épreuve qui enchaîne chacun à son existence. L'impossibilité de fuir sa douleur et d'échapper à l'étau de sa souffrance s'inscrit dans la plainte, le cri, le sanglot, la bouche bée, ces aveux d'impuissance devant plus fort que soi, la mort. Dès ses premiers ouvrages, le philosophe enracine sa réflexion dans la phénoménologie de l'élémental, c'est-à-dire dans la vie comme jouissance et dans le travail comme peine et douleur. Jouissance et douleur ont une commune frontière ${ }^{26}$. À partir de ce lieu concret, Levinas affirme que la souffrance est inutile, puisqu'elle est un mal - une négation de l'heure bonne provoquant un enfermement en soi de l'individu qui la pâtit: "Non du mal, négatif jusqu'au non-sens. [...] Le mal de la douleur, la nuisance même, est l'éclatement et comme l'articulation la plus profonde de l'absurdité. Que dans son phénomène propre, intrinsèquement, la souffrance soit inutile, qu'elle soit "pour rien", est donc le moins qu'on puisse en dire» (Levinas, 1984, p. 330). Rappelons que, à l'instar du langage courant, le philosophe emploie souvent l'un pour l'autre les termes douleur et souffrance. Mais il en élargit le registre à tout ce qui fait «souffrir», dénonçant comme particulièrement inutile et absurde la souffrance que l'être humain inflige de mille manières à son semblable.

Absurde et inutile est la souffrance, certes, mais Levinas découvre dans «la souffrance pour réduire la souffrance d'autrui» la seule justification de ce phénomène et l'expression d'une particularité qui fait la dignité de l'homme. "Autant sa propre douleur est inassumable, dit-il, autant on peut assumer dans une certaine mesure celle d'autrui, et en tout cas, on ne peut pas y rester indifférent. La non-indifférence à l'autre et à sa douleur [...] est la modalité première de la socialité avant toute convivialité» (Levinas, 1994, p. 133). Un prisonnier ne peut pas sortir seul de son enfermement. La compassion est une main tendue à l'autre: "la souffrance pour la souffrance inutile de l'autre homme, la juste souffrance en moi pour la souffrance injustifiable d'autrui, ouvre sur la souffrance la perspective de l'inter-humain » (Levinas, 1984, p. 331).

L'inter-humain se révèle dans le recours des uns au secours des autres et dans la responsabilité des uns pour les autres, antérieure à toute recherche de réciprocité qui annulerait l'altruisme, le désintéressement. Personne, répète Levinas, ne peut laisser l'autre homme souffrir et mourir seul. Envisagée dans une perspective inter-humaine, la souffrance, si elle est inutile en autrui, devient sensée dans celui qui porte secours, car tout geste compatissant la restitue aux dimensions du sens: consolation et soutien opèrent une ouverture qui libèrent autrui souffrant de l'étau du «soi-même». En bref, la compassion confère à la souffrance «inutile» le sens qui fait défaut à tout mal. «Le fait qu'autrui puisse compatir à la souffrance de l'autre, est le grand événement humain, le grand événement ontologique. [...] c'est un signe de la folie humaine, inconnue des animaux. [...] L'amour d'autrui est d'abord la souffrance pour la souffrance de l'autre» (Levinas, 1994, p. 135).

Est-il quelqu'un pour rêver qu'un jour la science fabrique un «homme non souffrant, non mourant, non désirant» (Vasse, 1983, p. 35), un homme parfait et immortel, celui du paradis perdu qui semble avoir laissé des traces dans un certain imaginaire collectif? Le droit de ne pas souffrir ne saurait s'appliquer au champ entier de la souffrance. Celle-ci est liée à "l'irrémissible poids de l'être » et à l'engagement de l'individu dans l'existence. Aussi le droit de ne pas souffrir n'est-il pas un droit absolu qui prendrait place dans une charte des droits et libertés. Mais ce droit existe et peut être revendiqué dans la sphère du soulagement de la douleur où l'art médical doit s'exercer le mieux possible, sans tomber dans les excès de la 
vision entièrement artificialiste dénoncée par Lucien Sève dans le texte cité ci-dessus. Tous les citoyens peuvent réclamer le droit d'être soulagés dans leur douleur et en défendre la cause. Il est regrettable que la douleur soit trop souvent traitée comme une question secondaire, un simple symptôme et non un mal en soi, que les préjugés contre la morphine soient encore profondément ancrés dans l'inconscient collectif, que la formation des étudiants en médecine soit déficiente à cet égard. D'autant plus que des douleurs mal contrôlées aggravent l'état du patient en affectant son système nerveux, ses émotions et rendent hypothétique sa guérison; qu'une douleur sourde, rongeante, lancinante, de symptôme qu'elle était au départ, devient elle-même une douleur-maladie.

Le soulagement de la douleur engage à la fois le travail des chercheurs, la responsabilité professionnelle et éthique des soignants, celle des institutions hospitalières, le système public de santé, la volonté politique des gouvernants.

\section{Bibliographie}

ABIVEN, Maurice (1994). «Au bout du chemin », dans Autrement, Paris, Éd. Autrement, série «Mutations», $\mathrm{n}^{\circ} 142$.

BEAUCHAMP, Paul (2000). Cinquante portraits bibliques, Paris, Éd. du Seuil.

CADORÉ, Bruno (1994). L'expérience bioéthique de la responsabilité, Montréal, Artel/ Fides, coll. «Catalyses».

CANGUILHEM, Georges (1966). Le normal et le pathologique, Paris, Presses universitaires de France, coll. "Quadrige».

CÔTÉ, Marc-André (2000). "Insoutenable douleur», dans Le Devoir, 18-19 novembre 2000 cahier "Société et Santé».

FARRACHI, Armand (2001). «Pitié pour la condition animale», dans Le Monde diplomatique (avril).

GADAMER, Hans-Georg (1998). Philosophie de la santé, Paris, Grasset-Mollat.

GOOD, Byron (1998). Comment faire de l'anthropologie médicale? Médecine, réalité et vécu, Le Plessis-Robinson, Institut Synthélabo, coll. «Les empêcheurs de penser en rond».

GUILLEBAUD, Jean-Claude (2001). Le Principe d'humanité, Paris, Éd. du Seuil.

HOBBES, Thomas (1997). De la nature humaine, Paris, Éd. Actes Sud, coll. «Babel», $\mathrm{n}^{\circ} 292$.

JACOB, André (1998). L'Homme et le Mal, Paris, Éd. du Cerf, coll. «Humanités».

JACOBI, Benjamin (1994). «La plainte», dans Autrement, Paris, Éd. Autrement, série «Mutations», no 142.

KEMP, Peter (1997). L'irremplaçable. Une éthique de la technologie, Paris, Cerf, coll. «Passages».

LEVINAS, Emmanuel (1994). «Une éthique de la souffrance», Entretien de Jean-Marc
Noris avec Levinas, dans Autrement, Paris, Éd. Autrement, série «Mutations», $\mathrm{n}^{\circ} 142$.

LEVINAS, Emmanuel (1984). «La souffrance inutile», dans Les Cahiers de la Nuit Surveillée. Emmanuel Levinas, $\mathrm{n}^{\circ} 3$, Lagrasse, Éd. Verdier, p. 329-338.

LEVINAS, Emmanuel (1983). Le temps et l'autre, Paris, Presses universitaires de France, coll. "Quadrige».

LEVINAS, Emmanuel (1974). Autrement qu'être ou au-delà de l'essence, La Haye, M. Nijhoff.

MARCEL, Gabriel (1964). La dignité humaine et ses assises existentielles, Paris, Éd. Aubier.

MARCEL, Gabriel (1935). Être et Avoir, Paris, Éd. Montaigne.

MARET, Michel ((2000). L'euthanasie. Alternative sociale et enjeux pour l'éthique chrétienne, Saint-Maurice, Éd. Saint-Augustin.

MARQUEZ, Catherine (1994). «Le mal chronique», dans Autrement, Paris, Éd. Autrement, série «Mutations», $\mathrm{n}^{\circ} 142$.

MEIRE, Philippe (1999). "Perspectives médico-psychologiques. Souffrance du corps vécu ", dans Revue d'éthique et de théologie morale, Paris, Éd. du Cerf, no 209, p. 27-46.

MEYER, Philippe (2000). Philosophie de la médecine, Paris, Éd. Grasset, coll. «Le Collège de Philosophie».

MÜLLER, Denis et Hugues POLTIER (dir.) (2000). La dignité de l'animal. Quel statut pour les animaux à l'heure des technosciences?, Genève, Labor et Fides, coll. «Le champ éthique».

NANCY, Jean-Luc (1995). «La douleur existe, elle est injustifiable », dans Revue d'éthique et de théologie morale, $\mathrm{n}^{\circ} 195$, p. 91-96.

POHIER, Jacques (1998). La mort opportune. Les droits des vivants sur la fin de leur vie, Paris, Éd. du Seuil, coll. «Essais».

REY, Roseline (1994). "Avec ou sans douleurs: une conquête dans l'Histoire», dans Autrement, Paris, Éd. Autrement, série «Mutations», n ${ }^{\circ} 142$.

RICOEUR, Paul (1997). «L'Éthique, entre le Mal et le Pire», dans Christian HERVÉ (dir.), Éthique médicale ou bioéthique? Paris, L'Harmattan, coll. «L'Éthique en mouvement», p. 17-33.

RICOEUR, Paul (1996). "Les trois niveaux du jugement médical», dans Esprit, n 227.

RICOEUR, Paul (1995). La critique et la conviction. Entretien avec François Azouvi et Marc de Launay, Paris, Éd. Calmann-Lévy.

RICOEUR, Paul (1994). «La souffrance n'est pas la douleur ", dans Autrement, Paris, Éd. Autrement, série «Mutations», $\mathrm{n}^{\circ} 142$.

SAINT-GERMAIN, Christian (2001). La technologie médicale hors-limite. Le cas des xénogreffes, Sainte-Foy, Presses de l'Université du Québec.

SÈVE, Lucien (2001). "Réflexions autour de l'arrêt Perruche. Pour une éthique de la maîtrise sans emprise", dans Les Cahiers du Comité consultatif national d'éthique, $\mathrm{n}^{\circ} 29$, p. 13-15.
SIBONY, Daniel (1995). «La douleur», dans Revue d'éthique et de théologie morale, Paris, Éd. du Cerf, no 195, p. 97-105.

SICARD, Didier (1999). Hippocrate et le scanner. Réflexions sur la médecine contemporaine, Paris, Éd. Desclée de Brouwer, coll. «Esculape».

SINGLETON, Michaël (1994). «Du leurre de la douleur», dans Autrement, Paris, Éd. Autrement, série «Mutations», no 142.

THÉVENOT, Xavier (1997). Compter sur Dieu, Paris, Éd. du Cerf, coll. "Recherches morales. Positions».

VASSE, Denis (1983). Le poids du réel, la souffrance, Paris, Éd. du Seuil.

VERSPIEREN, Patrick (1998). «Médecine et soulagement de la souffrance humaine», dans le Laval théologique et philosophique, vol. 54, no 1, p. 23-39.

VOGEL, Peter (2000). «La douleur animale», dans Denis MÜLLER et Hugues POLTIER (dir.), La dignité de l'animal. Quel statut pour les animaux à l'heure des technosciences?, Genève, Labor et Fides, coll. "Le champ éthique», p. 281-295.

\section{NOTES}

1. «La souffrance est beaucoup plus vaste, plus globale, elle est existentielle; elle inclut les dimensions physique, psychologique, sociale et spirituelle, dimensions très fortement imbriquées. L'homme éprouve la douleur dans son corps, alors que la souffrance est éprouvée dans tout l'être. Néanmoins, la douleur ellemême n'est pas purement physique [...] La douleur et la souffrance se renforcent mutuellement: une douleur très forte et persistante peut influer sur toutes les dimensions de la souffrance; inversement, l'anxiété, la dépression, la solitude ou le sentiment du non-sens de la vie accentuent la douleur. Si bien que la douleur physique a elle-même des composantes psychologiques, sociales et spirituelles. » Michel Maret, L'euthanasie. Alternative sociale et enjeux pour l'éthique chrétienne. Saint-Maurice, Éd. Saint-Augustin, 2000, p. 222.

2. Cette définition est citée, entre autres, par Philippe Meyer, Philosophie de la médecine, Paris, Éd. Grasset, coll. «Le Collège de Philosophie», 2000, p. 377.

3. Dans son livre De la nature humaine, Thomas Hobbes attribue le plaisir et le déplaisir ressentis par un sujet à ce qui favorise ou contrarie le mouvement vital. Ainsi, les objets qui aident le mouvement vital procurent à l'individu du plaisir, du contentement, du bien-être et sont appelés bons; ceux qui l'affaiblissent ou l'arrêtent, génèrent de la douleur et de la haine et sont considérés comme un mal. «Cette sollicitation est un effort ou un commencement interne d'un mouvement animal qui se nomme appétit ou désir quand l'objet est agréable, qui se nomme aversion lorsque l'objet déplaît actuellement, et qui se nomme crainte relativement au déplaisir que l'on attend» (Paris, Éd. Actes Sud, coll. «Babel», 1997, p. 53).

4. Voir Jean-Luc Nancy, «La douleur existe, elle est injustifiable», dans Revue d'éthique et de théologie morale, $\mathrm{n}^{\circ} 195$, p. 92-93. 
5. Voir André Jacob, L'Homme et le Mal, Paris, Éd. du Cerf, coll. «Humanités», 1998: «on ne fait guère l'économie du corps dans la souffrance "morale" ou psychologique, puisque l'activité ou les sentiments qui la portent se réfèrent immanquablement à une individualité sensible, quelle que soit sa "subjectivation" complexe» (p. 26).

6. Emmanuel Levinas, Autrement qu'être ou au-delà de l'essence, La Haye, M. Nijhoff, 1974, p. 71. Le philosophe Jean-Luc Nancy rejoint Levinas en disant que la jouissance est une douleur qui se perd dans la jouissance. L'une et l'autre se touchent en leurs extrémités (loc. cit., p. 96). Paul Ricoeur note un point qui leur est commun: "La souffrance est, avec la jouissance, la retraite ultime de la singularité.» (Voir "Les trois niveaux du jugement médical», dans Esprit, n $^{\circ} 227$, 1996, p. 22.) Denis Vasse place la souffrance et la joie «sur le même axe: celui de la vérité du désir dont l'authenticité n'est référable à aucun paramètre dans l'ordre de l'imaginaire. La souffrance comme la joie nous tirent de la fascination du même» (Le poids de la souffrance... p. 73-74).

7. Le cinéaste Hugo Latulippe, dans Bacon - le Film, produit par l'Office national du film, 2001 , en fournit un bon exemple.

8. La progression des sciences tant génétiques que cognitives invite malheureusement un certain nombre de penseurs à grignoter la frontière que la tradition a toujours placée et respectée entre l'homme et l'animal: les molécules d'ADN et d'ARN qui constituent le code génétique des deux espèces ont, sinon une parfaite identité, tout au moins une grande similitude; cette découverte encourage, non sans risque, la pratique médicale des xénogreffes. En outre, les primates supérieurs manifestent des aptitudes à l'apprentissage d'une certaine forme de langage. Les primatologues observent chez eux l'expression de sentiments qu'ils assimilent à la joie ou à la compassion. Entre l'espèce humaine et animale, on en vient à parler, non plus de frontière, mais de seuil de complexité, en particulier de cette complexité qui concerne la vie mentale. Il n'est pas de mon propos de discuter ces sujets, ni de développer une réflexion sur la douleur des animaux. Pour une plus ample réflexion, voir Denis Müller et Hugues Poltier, dir., La dignité de l'animal. Quel statut pour les animaux à l'heure des technosciences?, Genève, Labor et Fides, coll. "Le champ éthique», 2000. Voir aussi JeanClaude Guillebaud, Le Principe d'humanité, Paris, Éd. du Seuil, 2001, p. 43-70; Armand Farrachi, "Pitié pour la condition animale», dans Le Monde diplomatique, avril 2001, p. 21; Christian Saint-Germain, La technologie médicale hors-limite. Le cas des xénogreffes, Sainte-Foy, Presses de l'Université du Québec, 2001.

9. L'anthropologue Michaël Singleton affirme qu'il y a autant de perceptions de la douleur qu'il existe de sociétés. Il raconte son expérience déroutante chez les Wakonongo, au sud-ouest de la Tanzanie. Il a découvert que, dans cette culture, la douleur est toujours fonction d'un malaise social: qui laisse le corps social en souffrance ne viendra jamais à bout de la douleur corporelle. Voir son article «Du leurre de la douleur», dans Autrement, Paris, Éd. Autrement, série «Mutations», $n^{\circ} 142$, (1994), p. 152-161.

10. Roselyne Rey en explique la cause: la douleur aiguë «ne subsiste qu'en se nourrissant de l'énergie du corps et [...] celui-ci a besoin de moments d'accalmie pour sentir de nouveau et souffrir encore: l'évanouissement, la syncope $[\ldots]$ sont comme le signe de cette limite biologique qui, au-delà d'un certain seuil d'intensité, dérobe tout à la fois le corps et la conscience de l'individu qui subit la douleur physique». Voir l'article "Avec ou sans douleurs: une conquête dans l'Histoire», dans Autrement, loc. cit., p. 75.

11. Voir Peter Vogel, «La douleur animale», dans Denis Müller et Hugues Poltier, La dignité de l'animal, op. cit.: "les personnes souffrant d'absence de douleur sont couvertes de blessures, dès lors qu'il leur manque des signaux d'avertissement du danger. Bien qu'elles apprennent à éviter les coups, ces personnes souffrent finalement de dégénération des articulations et de la colonne vertébrale, car elles ne décèlent pas les limites de charge tolérable pour leurs organes. Dégénération et infections conduisent finalement à une mort précoce » (p. 283).

12. Emmanuel Levinas évoque ces douleursmaladies «auxquelles l'intégration aux autres états psychologiques n'apporte aucun soulagement, mais où, au contraire, l'angoisse et la détresse ajoutent à la cruauté du mal». Il fait aussi référence aux douleurs-maladies des êtres psychiquement déshérités, arriérés, diminués dans leur vie de relations et dans leurs rapports avec autrui, et à la «malignité sauvage » de cette souffrance. Voir «La souffrance inutile», dans Les Cahiers de la Nuit Surveillée. Emmanuel Levinas, no 3, Lagrasse, Éd. Verdier, 1984, p. 331.

13. Paul Beauchamp se demande s'il n'y a pas un rapport entre la maladie et le mensonge. Car le mensonge, qu'il s'adresse aux autres ou à soi-même, est un poison. Voir Cinquante portraits bibliques, Paris, Éd. du Seuil, 2000, p. 82.

14. Voir Daniel Sibony, «La douleur», dans Revue d'éthique et de théologie morale, $\mathrm{n}^{\circ} 195$, (p. 102-103): "Monsieur, la médecine ne peut rien pour vous; les lois du savoir restent muettes sur votre cas. Mais curieusement on en déduit: "c'est dans votre tête que ça se passe". Comme si le savoir - incarné par la médecine ou par autre chose - couvrait à tel point le corps, que s'il ne peut rien lui dire, c'est que l'affaire a lieu dans le brouillard de la parole. (En outre, pourquoi ce qui "se passe dans la tête" ferait-il moins mal?».

15.Ibid., p. 97.

16. Citons ad hoc Catherine Marquez: «Le sujet transforme la douleur suivant sa personnalité et les aléas de son état actuel. [...] Par le jeu de chaînes associatives sont réactivées les frustrations, la culpabilité, le désir de punition, la douleur des séparations, l'agressivité qu'il n'a pas pu exprimer, les sentiments de devoir payer une faute commise par lui, voire par un autre» (loc. cit., p. 38).
17. Voir Xavier Thévenot, Compter sur Dieu, Paris, Éd. du Cerf, coll. «Recherches morales. Positions », 1997, chapitre VI, particulièrement p. 130, à propos de la dysphorie temporelle.

18. André Jacob, L'Homme et le Mal, op. cit., p. 20. Levinas dit lui aussi que la souffrance "est une impossibilité de se détacher de l'instant de l'existence». Le temps et l'autre, op. cit., p. 55.

19. Gabriel Marcel la compare quelque part à un malade alité incapable de changer de position. Levinas la qualifie d'inassumable.

20.Jean-Luc Nancy, loc. cit., p. 95. Ce que dit l'auteur à propos de la douleur vaut pour la souffrance, puisque l'une et l'autre en viennent à se fondre dans un même mal-aise.

21. Voir, entre autres, Gabriel Marcel, La dignité humaine et ses assises existentielles, Paris, Éd. Aubier, 1964, p. 141-142, où il analyse sa pièce de théâtre, La Chapelle ardente, et montre même l'effet «toxique» de la souffrance chez le personnage principal.

22. Quelqu'un peut trouver un sens à sa propre souffrance, mais jamais se permettre d'en attribuer un à celle des autres, disait Gabriel Marcel.

23. Voir Gabriel Marcel, Etre et Avoir, Paris, Éd. Montaigne, 1935, p. 207-209. Je rapprocherai l'intuition de Marcel d'une réflexion de Paul Ricoeur qui, se référant au suicide de son fils Olivier, l'enfant du retour de captivité, l'enfant de la paix, dit à ses interlocuteurs: «Je rejoignais ainsi le lot immense de tant de pères et découvrais cette fraternité silencieuse qui naît de l'égalité dans la souffrance.» (La critique et la conviction. Entretien avec François Azouvi et Marc de Launay, Paris, Éd. Calmann-Lévy, 1995, p. 141.)

24.Benjamin Jacobi, "La plainte», dans Autrement, loc. cit., p. 118: «C'est la rupture de la naissance qui entremêle cris et plaintes, douleur de l'expulsion et souffrance de la séparation. Ce sont les séparations de la mère dans la vie quotidienne, dans le sevrage progressif qui alimenteront les premiers cris de l'enfant et annonceront son entrée dans le symbolique quand il parviendra à appeler: "maman"». Selon cet auteur, la sollicitation du soignant et sa sollicitude «réactivent dans le lien soignant-soigné des représentations maternelles inconscientes» (p. 120). Il est intéressant de rapprocher ce point de vue de celui de Levinas qui voit dans le pleur et le sanglot une inversion de la souffrance sur elle-même. Et il ajoute: «là où la souffrance atteint à sa pureté, où il n'y a plus rien entre nous et elle, la suprême responsabilité de cette assomption extrême tourne en suprême irresponsabilité, en enfance. C'est cela le sanglot et par là précisément il annonce la mort. Mourir, c'est revenir à cet état d'irresponsabilité, c'est être la secousse enfantine du sanglot». ( $L e$ temps et l'autre, op. cit., p. 60.)

25.Xavier Thévenot, op. cit., p. 129. Nous renvoyons le lecteur au chapitre VI: «La compassion: une réponse au mal? » qui éclaire les ambiguités dont cette attitude est porteuse. Ce chapitre ne peut ici que m'inspirer.

26. Voir ci-dessus, note 6 . 